\title{
A novel large deletion of the ICR1 region including H19 and putative enhancer elements
}

\author{
Helen Fryssira ${ }^{1 *}$, Stella Amenta ${ }^{2}$, Deniz Kanber ${ }^{3}$, Christalena Sofocleous ${ }^{1}$, Evangelia Lykopoulou ${ }^{4}$, \\ Christina Kanaka-Gantenbein ${ }^{4}$, Flavia Cerrato ${ }^{5}$, Hermann-Josef Lüdecke ${ }^{3}$, Susanne Bens ${ }^{6}$, Andrea Riccio ${ }^{5}$ \\ and Karin Buiting ${ }^{3}$
}

\begin{abstract}
Background: Beckwith-Wiedemann syndrome (BWS) is a rare pediatric overgrowth disorder with a variable clinical phenotype caused by deregulation affecting imprinted genes in the chromosomal region 11p15. Alterations of the imprinting control region 1 (ICR1) at the IGF2/H19 locus resulting in biallelic expression of IGF2 and biallelic silencing of $\mathrm{H} 19$ account for approximately $10 \%$ of patients with BWS. The majority of these patients have epimutations of the ICR1 without detectable DNA sequence changes. Only a few patients were found to have deletions. Most of these deletions are small affecting different parts of the ICR1 differentially methylated region (ICR1-DMR) removing target sequences for CTCF. Only a very few deletions reported so far include the H19 gene in addition to the CTCF binding sites. None of these deletions include IGF2.
\end{abstract}

Case presentation: A male patient was born with hypotonia, facial dysmorphisms and hypoglycemia suggestive of Beckwith-Wiedemann syndrome. Using methylation-specific (MS)-MLPA (Multiplex ligation-dependent probe amplification) we have identified a maternally inherited large deletion of the ICR1 region in a patient and his mother. The deletion results in a variable clinical expression with a classical BWS in the mother and a more severe presentation of BWS in her son. By genome-wide SNP array analysis the deletion was found to span 100 kb genomic DNA including the ICR1DMR, H19, two adjacent non-imprinted genes and two of three predicted enhancer elements downstream to H19. Methylation analysis by deep bisulfite next generation sequencing revealed hypermethylation of the maternal allele at the IGF2 locus in both, mother and child, although IGF2 is not affected by the deletion.

Conclusions: We here report on a novel large familial deletion of the ICR1 region in a BWS family. Due to the deletion of the ICR1-DMR CTCF binding cannot take place and the residual enhancer elements have access to the IGF2 promoters. The aberrant methylation (hypermethylation) of the maternal IGF2 allele in both affected family members may reflect the active state of the normally silenced maternal IGF2 copy and can be a consequence of the deletion. The deletion results in a variable clinical phenotype and expression.

Keywords: Beckwith-Wiedemann syndrome, Genomic imprinting, Imprinting disorders, DNA methylation

\section{Background}

Beckwith-Wiedemann syndrome (OMIM 130650) is an overgrowth disorder characterized by exomphalos/omphalocele, macroglossia, gigantism and high risk for tumor development [1-3]. BWS has an estimated pan-ethnic incidence of $1 / 13,700$. It is usually sporadically transmitted (85\%), but familial occurrence has been reported in about

\footnotetext{
* Correspondence: efrysira@yahoo.gr

'Aghia Sophia Children's Hospital, University of Athens Medical School, Athens, Goudi 11527, Greece

Full list of author information is available at the end of the article
}

$15 \%$ of cases [3]. Variations in clinical manifestations may be linked to molecular heterogeneity [4]. Different and complex molecular mechanisms have been implicated in the dysregulation of imprinted growth regulatory genes on chromosome 11p15 [5].

Evidence of three major or two major and three minor criteria usually supports the diagnosis of BWS. Major clinical findings includes among others: macrosomia, macroglossia, omphalocele or umbilical hernia, ear pits or anterior linear ear lobe creases, visceromegaly, adrenocortical cytomegaly, hemihyperplasia, renal abnormalities, 
and embryonal tumors. Minor signs are: polyhydramnios, neonatal hypoglycemia, characteristic facies, advanced bone age, naevus flammeus, cleft palate, rectus diastesis and cardiac anomalies [6].

Genomic imprinting is an epigenetic mechanism of gene modification, starting during gametogenesis and resulting in the differential expression of the paternal and maternal alleles during development. By this process, genes which are subject to genomic imprinting are expressed from one parental allele only. Deregulation of imprinted genes can lead to specific diseases. BWS syndrome is one of the most well-known human diseases implicated in genomic imprinting [7].

The 11p15.5 region contains two clusters of imprinted genes, which play a role in cell cycle and growth regulation $[8,9]$. Imprinted expression of these two gene clusters are regulated by two independent imprinting control regions, ICR1 and ICR2. ICR1 regulates the imprinted expression of genes in the most telomeric imprinted region including the insulin like growth factor 2 (IGF2) and H19. Molecular alterations affecting the ICR1 in patients with BWS lead to biallelic expression of IGF2 and to loss of function of H19. ICR2 controls the imprinted expression of genes in the more centromeric region, such as cyclindependent kinase inhibitor $1 \mathrm{C}(C D K N 1 C / p 57)$, potassium voltage-gated channel subfamily Q member 1 (KCNQ1) and KCNQ1- overlapping transcript 1 (KCNQ1OT1 or LIT1) [8,9]. Molecular alterations in BWS affecting the ICR2 lead to silencing of $C D K N 1 C$ and $K C N Q 1$ and biallelic expression of KCNQ1OT1. At least one of the many genetic/epigenetic alterations is recognized in 80\% of cases [9] and includes the following:

- Isolated hypomethylation of the KvDMR/ICR2 ( $50-60 \%)$, which is implicated with the reduction of $C D K N 1 C / p 57$ expression

- Paternal uniparental isodisomy of 11p15.5 ( 20\%)

- Isolated gain of methylation of ICR1 and biallelic IGF2 expression (2-7\%)

- Chromosome rearrangements $(\operatorname{dup}(11)(\mathrm{p} 15)$ of paternal origin) (1-2\%)

- Maternal CDKN1C mutations, representing 5-10\% of sporadic cases and $4050 \%$ of familial cases

- Rare cases with maternal deletions affecting the ICR1 or ICR2 (less than 1\%)

- Rare cases with small deletions or mutations affecting an OCT4 binding motif inside the ICR1-DMR between $H 19$ and IGF2.

Familial or de novo deletions in BWS affecting the ICR1 or ICR2 on chromosome $11 \mathrm{p} 15$ are rare findings. Only four maternal deletions affecting the ICR2 have been reported so far [10-13] However, in one case the deletion includes the $C D K N 1 C$ gene, explaining the BWS phenotype because of the lack of the maternal CDKN1C transcript. All these ICR2 deletions are large in size spanning 198$900 \mathrm{~kb}$ genomic DNA. In contrast, most of the ICR1 deletions identified so far, are small microdeletions of 0.5 to $2.8 \mathrm{~kb}$ affecting CTCF binding inside the ICR1 [14-21]. For some of these deletions (sized $1.4 \mathrm{~kb}, 1.8 \mathrm{~kb}$ and 2.2 $\mathrm{kb}$, respectively) it has been shown that the phenotypical outcome appears to depend on the spatial arrangement of the remaining CTCF-binding sites [21]. Only a few larger maternally inherited deletions in patients with BWS including $H 19$ in addition to the ICR1 have been described recently by Baskin et al. [19] and one paternally inherited deletion in a mosaic state, which interestingly lead to atypical Silver-Russel syndrome (SRS, an intrauterine growth retardation syndrome) $[22,23]$.

Here, we present a novel familial case of BWS, in which the underlying molecular defect is a rare maternally transmitted deletion of $\sim 100 \mathrm{~kb}$ in size. The deletion was found to be on the maternal chromosome 11 in the mother and her son. Both have BWS, although the clinical phenotype of the son was more severe than that of the mother. Hypermethylation of the DMR0 of IGF2 in both affected family members, gives a hint for biallelic expression of $I G F 2$, which is causative for the BWS phenotype.

\section{Case presentation}

The male patient of Greek origin was the only child of the parents. He was born after 40 weeks of gestation through a caesarian section due to breech presentation [birth weight $3.250\left(50^{\text {th }}\right.$ centile), length $53.5 \mathrm{~cm}\left(>95^{\text {th }}\right.$ centile), head circumference $34.6 \mathrm{~cm}$ (50 ${ }^{\text {th }}$ centile)]. The Apgar score was 8 at $1^{\prime}$ and 9 at $5^{\prime}$. The parents were nonconsanguineous (mother was 35 and father was 36 years old) and the conception was not assisted. The pregnancy and the prenatal ultrasound were reported as normal. The mother of the child had macrosomia (height $=95^{\text {th }}$ centile), large hands and feet with lymphedema of the lower limbs. The mother's phenotype from her childhood was suggestive of the clinical diagnosis of BWS (gigantism, macroglossia and small omphalocele). The patient's father, the maternal grandmother and grandfather were reported as phenotypically normal.

At birth, the newborn was hypotonic with facial BWS dysmorphisms, macrosomia but with no obvious omphalocele. There was a small palpable mass at the sacrococcygeal region (Figure 1A, B, C). In the first 24 hours, the patient presented with hypoglycemia attributed to hyperinsulinism and was treated with diazoxide. The hypoglycemia was persistent and intractable. From the biochemical screening, in addition to hypoglycemia, the patient had AFP (60.500 ng/ml-normal range maximum 40.000), liver function tests (transaminases) and direct bilirubin levels which were remarkably elevated, but with 


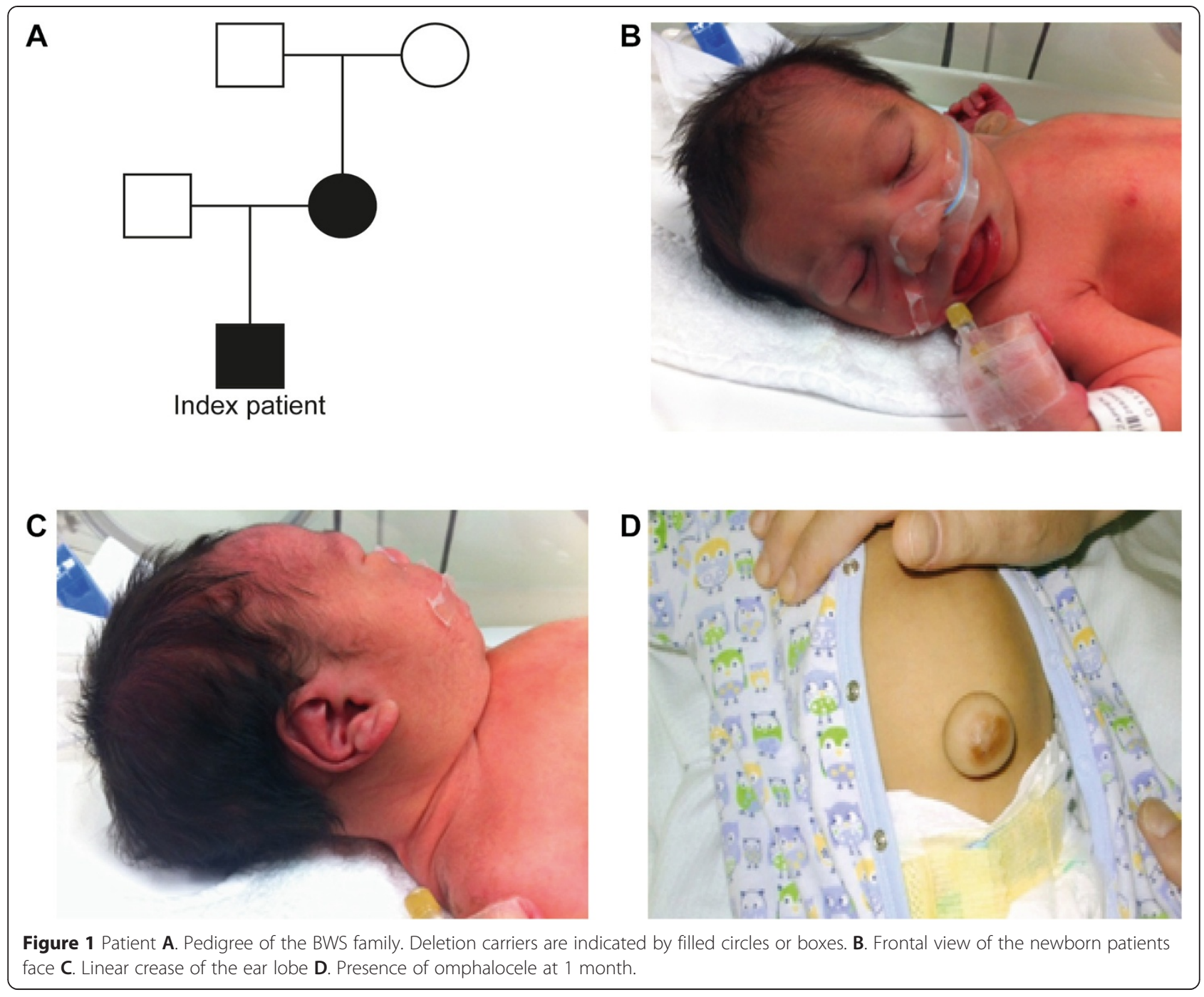

normal ammonia levels. The cardiac ultrasound revealed an atrial septal defect with mild hypertrophy of the septum and the right ventricle, along with mild bilateral stenosis of the pulmonary arteries. TORCH and metabolic investigations were negative. Due to the intractable hypoglycemia, a therapeutic trial with somatostatin was also attempted, but without relevant clinical benefit. At the age of two months, the proband was still mildly hypotonic, had macrosomia, a notable umbilical hernia, macroglossia, and hypertelorism with a flat nasal bridge, all of which are clinical features of BWS. Heart ultrasound of the patient remained the same as during the first 24 hours. Abdominal ultrasound and Magnetic Resonance Imaging (MRI) confirmed the hepatomegaly but without dilatation of the intra and extrahepatic biliary vessels, the splenomegaly, a normal pancreas, adrenals and enlarged but otherwise morphologically normal kidneys. An X-ray of the left hand, also taken at two months, revealed advanced bone age (one year more than his chronological age). Due to the presence of a small sacrococcygeal mass, MRI of the lower spine revealed spina bifida at the level of $I_{1}-I_{2}$ vertebra. Because of the macroglossia, the patient developed severe upper airway congestion and was placed on mechanical ventilation (CPAP). After eight days his blood sugar stabilized. After 5 months of hospitalization, his AFP levels dropped and he could eat and breathe by himself. After clinical reevaluation at the ages of 8,12 and 15 months his macrosomia remained out of the $97^{\text {th }}$ centile (above 2 SDs). At 15 months his developmental milestones were slightly delayed (he walks with support) but he did not present a neurological deficit.

\section{Cytogenetic analysis}

Conventional karyotyping by G-banding technique was carried out on stimulated peripheral blood cultures on metaphase cells according to standard protocol. Fifty metaphases with 550 band resolution $(\geq 5 \mathrm{Mb})$ were analyzed. 


\section{DNA extraction}

Genomic DNA from peripheral blood was extracted according to standard protocols (QIAGEN BioRobot M48, MagAttract DNA Blood Kit, Hilden, Germany).

\section{Methylation-specific multiplex ligation-dependent probe amplification (MS-MLPA)}

Gene dosage and methylation of the H19/ICR1-DMR/ IGF2 and ICR 2 on chromosome $11 \mathrm{p} 15$ were analyzed by MS-MLPA using the SALSA MLPA KIT ME030-C3 BWS/RSS (MRC Holland, Amsterdam, Netherlands). Hybridization, ligation and PCR reactions were carried out according to the manufacturer's instructions. Amplification products were analyzed by capillary electrophoresis using the ABI3100 or 3500 capillary sequencer, respectively. Data analysis was carried out using the Gene Marker Software (Softgenetics, State College, PA, USA).

\section{Bisulfite treatment of DNA}

Bisulfite treatment of genomic DNA was carried out using the EZ DNA MethylationGold Kit (Zymo Research Europe, Freiburg, Germany) according to the manufacturer's protocol.

\section{Deep bisulfite sequencing}

Generation of bisulfite amplicon libraries, sample preparation and sequencing on the Roche 454 GS junior system was carried out as previously described $[21,24]$. For the IGF2 DMR0 following primers were used (product size $349 \mathrm{bp} ; \mathrm{Tm}=62^{\circ} \mathrm{C}$ ): IGF2-DMR0-Ftag $5^{\prime}$-CTTGC TTCCTGGCACGAG-AGATTTTTTTGTTGGATAGG 'TTGTT-3', IGF2-DMR0-RM13 5' - CAGGAAACAGCT ATGACTCTATTACACCCTAAACCCAAACTC -3' (in bold universal tags). For allele separation we used the SNP rs3741210 (A/G). For data analysis, we used the Python-based amplikyzer software developed in-house [25].

\section{Molecular karyotyping}

Array analysis was carried out using the genome-wide high-resolution SNP array CytoScan HD (Affymetrix) according to the manufacturer's protocols (Affymetrix, SantaClara, California, USA). Evaluation of the data was performed using the Affymetrix Chromosome Analysis Suite version 2.0.

\section{In silico enhancer characterization}

Candidate enhancer sequences of the IGF2/H19 genes have been identified through bioinformatics analysis for the presence of the following characteristics: 1) conserved non coding regions of at least $100 \mathrm{bp}$ ( $>70 \%$ identical between human and rodents); 2) enriched histone H3K27 acetylation and H3K4 monomethylation; 3) DNase hypersensitive sites and transcription factor binding [26-28].

\section{Results}

Cytogenetic analysis had revealed a normal karyotype in both, the mother and the patient. Because of the clinical diagnosis of BWS in the propositus and his mother, a combined gene dosage and methylation analysis by MSMLPA for the chromosome 11p15 imprinted region was performed in both. In addition, the father of the propositus and the maternal grandparents were investigated by MS-MLPA. By this method, a deletion affecting all nine of the ICR1-DMR and H19 specific MLPA probes was detected in the patient and his mother. All four methylationspecific MLPA probes for the H19/ICR1-DMR showed complete methylation in both affected individuals An additional excel file presents detailed MLPA findings (see Additional file 1: Table S1). In contrast, all other studied family members showed a normal MLPA pattern. The aberrant methylation pattern in both affected individuals indicated that both have the deletion on their unmethylated, i.e. maternal chromosome explaining the BWS phenotype. None of the MLPA probes for IGF2 or the ICR2 showed a reduction in the gene dosage, indicating that the deletion is restricted to the ICR1-DMR, H19 and probably to the downstream region of H19. Since the maternal grandmother has no deletion (data not shown), the deletion in the mother seems to be a de novo event; although a germline mosaicism for a deletion in the grandmother cannot be excluded.

To further narrow down the breakpoints of the identified deletion in $11 \mathrm{p}$, we performed genome wide array analysis in the patient. By this approach we determined the size of the deletion to at least $99.78 \mathrm{~kb}$ and at most $100.02 \mathrm{~kb}$. The deletion was described as arr[hg19] 11p15.5 $(1,965,037 \times 2$, $1,965,0472,064,827 \times 1,2,065,052 \times 2)$. The deletion encompasses the H19, miR-675, the ICR1DMR and the adjacent non-imprinted genes MRPL23, MRPL23-AS1, but not IGF2 or the ICR2 region (Figure 2). The centromeric breakpoint is estimated to map $\sim 52 \mathrm{~kb}$ downstream of H19. The telomeric breakpoint maps approximately $46 \mathrm{~kb}$ upstream of $H 19$ and therefore $90 \mathrm{~kb}$ downstream of IGF2.

Mouse transgenic studies have identified multiple shared enhancers for Igf 2 and $H 19$ between 10 and $120 \mathrm{~kb} \mathrm{3'}$ of $H 19$ [29]. Enhancer sequences are characterized by conserved non coding regions that are enriched in specific histone modifications (i.e. H3K27acetylation, H3K4monomethylation), DNase hypersensitive sites and transcription factor binding $[28,30]$. We identified four sequences meeting these features at the IGF2/H19 locus (Figure 2). Three of them are located 85, 26 and $10 \mathrm{~kb}$ at the $3^{\prime}$ of $H 19$, respectively, reflecting the position of murine enhancers that have been identified by transgenic mouse studies [29]. A fourth region with enhancer features is located in the intergenic region between IGF2 and H19, at $\sim 100 \mathrm{~kb}$ far from H19. Two of these sequences are located within the region deleted in our family. 


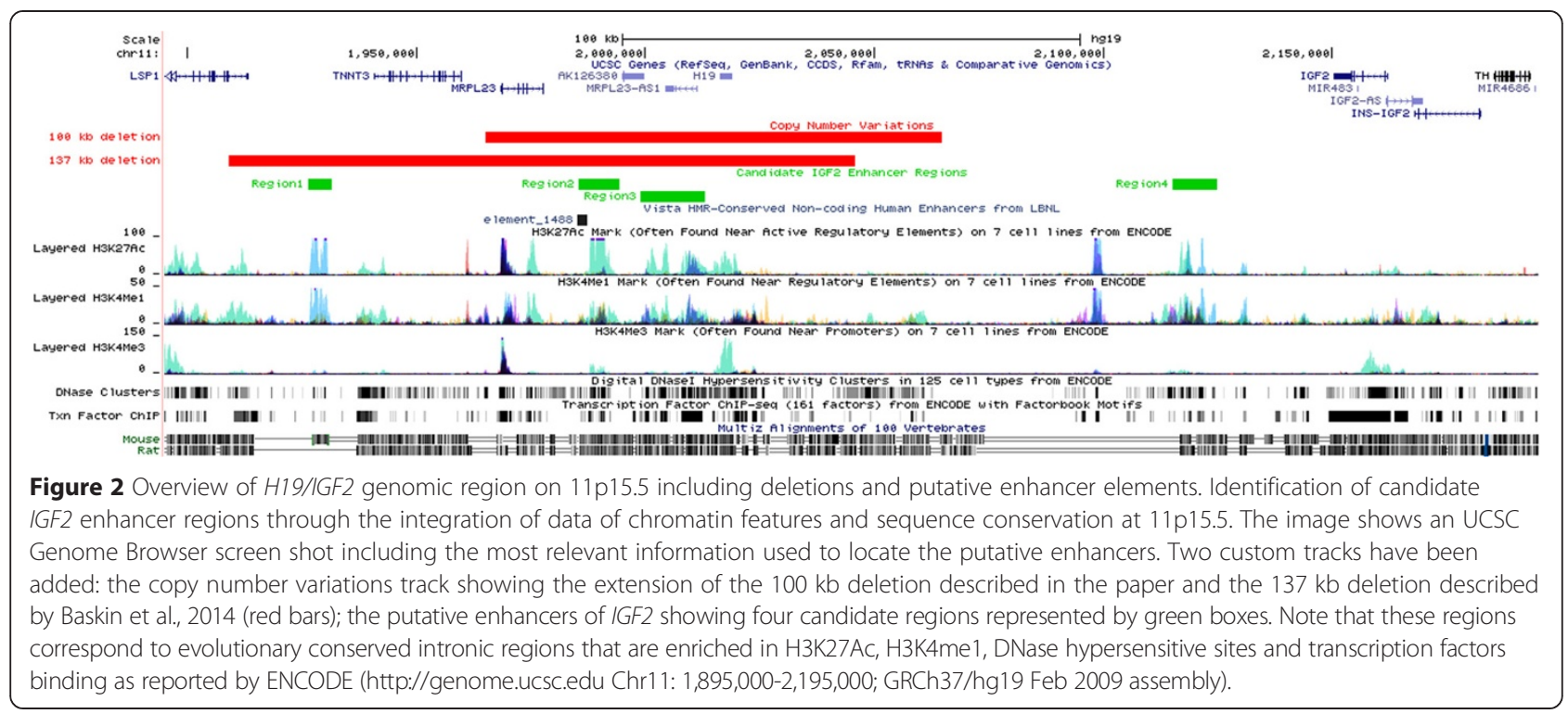

Since no probe for the IGF2 locus to test differential methylation was included in the MS-MLPA kit, we performed next generation bisulfite sequencing of the DMR0 of IGF2 in blood DNA from the mother, the father and the son and an unrelated normal control. Normally this DMR0 is hypermethylated $(\sim 70 \%)$ on the active paternal allele of IGF2 and hypomethylated ( 30\%) on the silent maternal allele [31] (and own unpublished data). We found a hypermethylation of the DMR0 in the mother and her son compared to the healthy father and the normal control. For the six CpG dinucleotides studied, the mother showed an average methylation of $71.7 \%$ and her son an average methylation of $70.8 \%$, while the average methylation in the father and the normal control (NC15) was $48.2 \%$ and $48.9 \%$, respectively (see Figure $3 \mathrm{~B}$ ). Since the patient and one of the normal controls were found to be heterozygous for a single nucleotide polymorphism (SNP, rs3741210, A > G) we were able to separate the parental alleles. Since the mother of the patient is homozygous for the G allele and the father is homozygous for the A allele, the parental origin of the two alleles in the patient could be determined. By this we could show that the normal control has one allele which is hypermethylated $(72.5 \%)$ whereas the other allele is hypomethylated (26.5\%, see Figure 3C). In contrast, in the patient both alleles (A and G) show a hypermethylation of $80.6 \%$ for the paternal allele (A) and $62.7 \%$ for the maternal allele (G), indicating that the maternal deletion allele acquired methylation at the DMR0 although the deletion does not overlap IGF2.

Our findings are in agreement with the results published by Murrell et al. [31].

\section{Conclusions}

BWS results from deregulation of imprinted genes on chromosome 11p15 and is known to have clinical variability and molecular heterogeneity. There are phenotypic differences between the various molecular subgroups of BWS [9]. Familial or de novo deletions affecting one of the two ICRs in 11p15 are a rare cause of BWS. Only 14 deletions for ICR1 have been described so far [14-21,23]. Ten of these deletions are very small and affect only the ICR1 itself. These deletions affect multiple target sites (CTSs) for the zinc-finger protein CTCF, whose binding on the maternal allele blocks the access of the IGF2 promoter to its downstream enhancers and enables H19 to use these enhancers for transcription. The ICR1-DMR deletions affect the ICR1 function and CTCF binding to different extents resulting in variable expression of the clinical phenotype depending on the spatial arrangement of the remaining CTSs [21]. Of the four larger deletions resulting in BWS after maternal transmission reported by Baskin et al. [19] three (18-25 kb) include the $H 19$ gene in addition to the ICR 1 and one deletion of $137 \mathrm{~kb}$ include the non-imprinted genes MRPL23, its antisense MRPL23-AS1, TNNT3 and part of the LSP1 gene downstream of $H 19$. However, none of these deletions include $I G F 2$. The deletion in our family is $\sim 100 \mathrm{~kb}$ in size and includes $H 19$, the miR-675, which is located inside $H 19$, the non-imprinted gene MRPL23, its antisense transcript MRPL23-AS1 and two of three putative enhancer elements downstream of $H 19$.

MRPL23 has previously been shown to be functionally insulated from the IGF2/ICR1/H19 domain in terms of both imprinting and enhancer action [32]. 
A

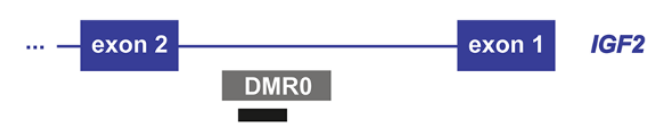

B

IGF2 DMRO

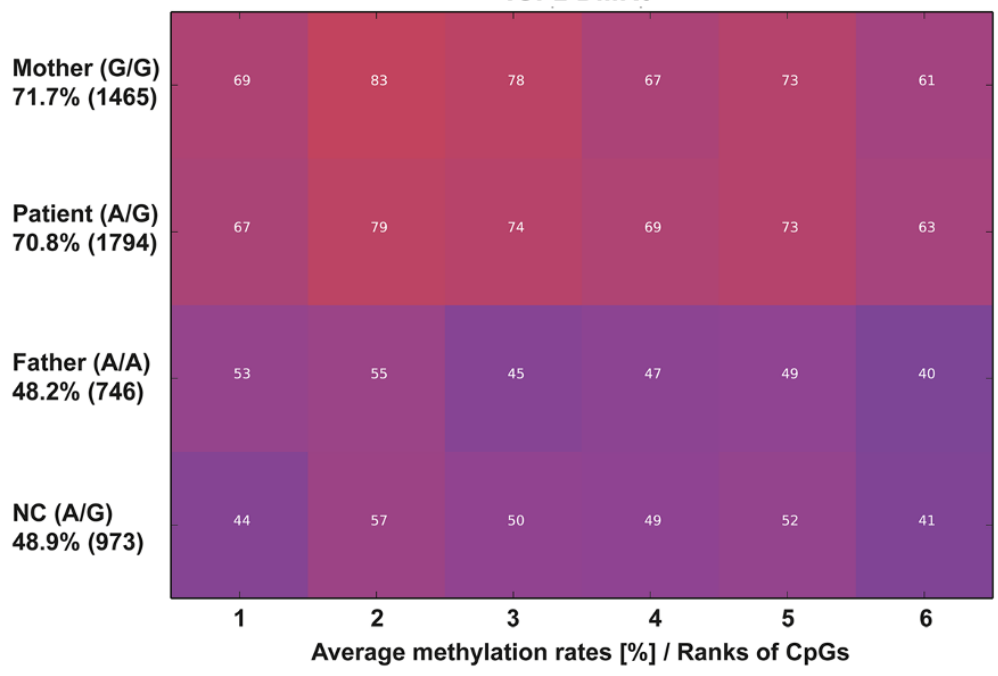

C

IGF2 DMRO

(alleles separated)

NC

Allele A

$72.5 \%$ (437)

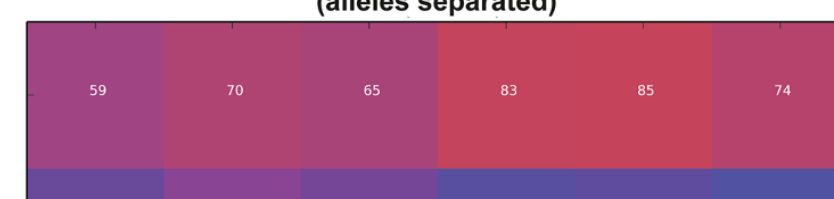

NC

Allele G

$26.5 \%$ (445)

Patient

Allele A

$80.6 \%(718)$

Patient

Allele G

$62.7 \%(813)$

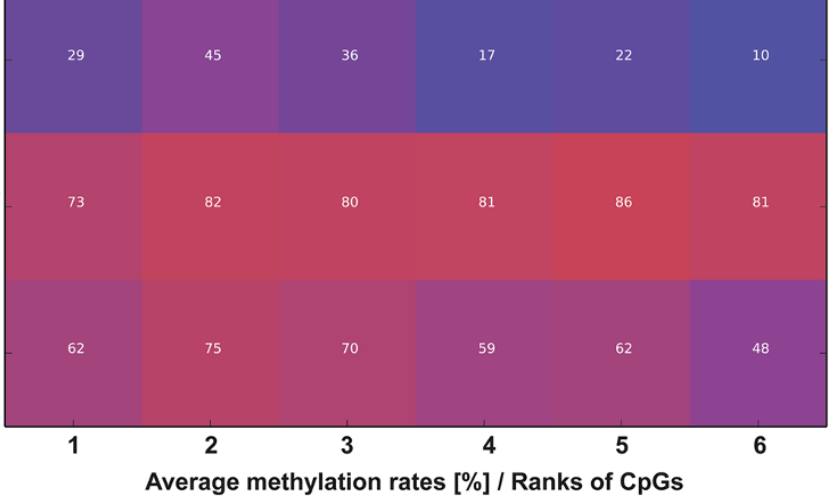

Figure 3 Comparative methylation plots and heatmaps for the IGF2 DMRO. (A) Schematic view of the IGF2 DMRO and the location of the amplicon used for deep bisulfite sequencing (black bar; not drawn to scale). The exact position for the amplicon is chr11:2169292-2169605 (hg19, UCSC Browser). (B) The average methylation of the IGF2 DMRO in the patient, his parents and a normal control. Compared to the father and the normal control (NC) the mother and the patient show a hypermethylation for the IGF2 DMRO. (C) The average methylation of the separated alleles (SNP rs3741210) in a normal control and the patient. One allele of the normal control is hypermethylated (indicating that it represents the paternal allele), whereas the other allele is hypomethylated (indicating that it representing the maternal allele). In the patient the paternal allele $(\mathbf{A})$ is hypermethylated and the maternal allele $(G)$, which should be hypomethylated, is hypermethylated as well. Each square represents a CpG dinucleotide with its average methylation level, each line a specific sample. The average methylation over the analyzed region is given in percentage on the left under the sample ID together with the number of analyzed reads in brackets. The methylation was analyzed over 6 CpGs.

Thus, a heterozygous deletion of MRPL23 should not give rise to any of the BWS phenotypic features present in the patient and the mother.

From mouse models but also from the deletion cases with small deletions inside the ICR1-DMR it is known that maternal transmission of a ICR1-DMR deletion itself leads to reactivation of the maternal IGF2 copy and therefore leads to biallelic expression of IGF2 resulting in BWS [14,20,29] Reactivation of IGF2 is associated with hypermethylation of the maternal allele [31]. We 
could demonstrate that the deletion leads to a gain of methylation of the maternal allele at the DMR0 of IGF2 in both, the patient and his mother. The DMR0 is located $5^{\prime}$ to the main promoters of IGF2. In 2008 Murrell and coworkers demonstrated that the IGF2-DMR0 is subjected to different methylation changes in cancer and congenital growth disorders [31]. In normal peripheral blood and kidney the DMR0 is predominantly methylated on the paternal allele and unmethylated on the maternal allele. In addition, BWS patients with and without microdeletions have IGF2-DMR0 as well as ICR1 hypermethylated while patients with Silver-Russell syndrome (SRS), a growth retardation syndrome, have hypomethylation at both of these sequences [31,33]. From these data it has been concluded that in early embryonic development the IGF2-DMR0 is methylated or unmethylated according to the germline methylation imprint of the ICR1-DMR. Gain of methylation of the DMR0 leads to activation of the maternal IGF2 allele in ICR1 hypermethylated BWS cases and loss of methylation of the DMR0 leads to silencing of the paternal IGF2 allele in ICR1 hypomethylated SRS cases [31].

In the family presented here, the deletion patient and his mother showed hypermethylation on the maternal deletion allele at the IGF2 DMR0, indicating that the maternal allele is transcriptionally active. In this case, the hypermethylation does not reflect the methylation status of the ICR1-DMR since this DMR is deleted. However, it may reflect the active state of the IGF2 promoters and possibly arises as a consequence of the interaction of the IGF2 promoters to its downstream enhancers. The exact location of these downstream enhancers is well known in mice but largely unknown in human [29]. By in silico analysis we could identify four candidate regions for putative enhancers. Two of these sequences are affected by the deletion in our family. From the methylation status of $I G F 2$ it seems that the residual two enhancer elements are sufficient to drive IGF2 expression on the maternal allele. Moreover, evidence that only the most telomeric and/or the most centromeric putative enhancer elements are needed to activate IGF2 comes from a patient with the only similar deletion of $137 \mathrm{~kb}$ in size reported by Baskin and colleagues [19]. Compared to our deletion, this deletion includes one additional putative enhancer element downstream of $H 19$ (see Figure 2). Although methylation or expression studies for IGF2 have not been performed in this patient, there must be an activation of the maternal IGF2 allele since the patient has phenotypic features of BWS. However, Gronskov et al. [23] recently reported an atypical SRS patient with a deletion in mosaic on the paternal chromosome 11, which is similar to the deletion in our patient. The telomeric breakpoint of this deletion is nearly the same whereas the centromeric breakpoint is closer to H19 and therefore spans also the same enhancer elements as the deletion in our patient. It is interesting that a deletion of $H 19$ and its enhancers, in opposite inheritance, can give rise to either atypical SRS or BWS. Furthermore, Gronskov et al. could show that in the SRS patient methylation of the IGF2 DMR0 is slightly changed to hypomethylation. The fact that aberrant methylation in this patient is not very strong may be due to the mosaic state of the deletion in this patient.

It is known that IGF2 has a key role in fetal and placental development by activating intracellular signaling cascades that promote cell growth and survival.

Interestingly, in a recent study it has been reported that the methylation level of IGF2DMR0 but not DMR2 is significantly increased in brain tissues of neural tube defect fetuses and that a hypermethylation of the IGF2DMR0 is positively associated with an increased risk of neural tube defects [34]. This might explain the presence of spina bifida in the lower back of our deletion patient, a feature which is not listed in the clinical features of BWS [34]. However, alterations affecting the ICR1-DMR and therefore in turn affecting IGF2 methylation and expression are only found in a minority of patients with BWS ( 10\%), making a judgment of increased spina bifida occurrence difficult. IGF2 upregulation is also known to be associated with an increased risk for embryonal tumors including Wilms tumor. Thus, the occurrence of embryonal tumors in early life is a major concern in patients with BWS and ICR1/IGF2 alterations. Our patient has not yet developed Wilms tumor but since he is at high risk for tumor development ultrasound surveillance is being performed in 3 month intervals until the age of 6 years.

Although the patient and his mother have the same deletion, the clinical phenotype of the patient is more severe than that of his mother. For example, he was severely affected by hyperinsulinaemic hypoglycemia which was not noted in his mother. A multidisciplinary approach was necessary to prevent the threat on the patient's life. It is not known how severely the patient with the $137 \mathrm{~kb}$ deletion [19] was affected, since clinical data for this patient have not been reported.

An increased clinical severity can be a random result of variable phenotypic expression, which is not uncommon in BWS. Evidence for anticipation in BWS in members of a family with a maternally inherited OCT4 binding site mutation has recently reported by Berland et al. [24]. Two sisters and their cousin had classical BWS with Wilms tumor whereas their mothers and a third sister of the mothers have only tall stature and only one of these sisters showed also mild BWS features as a child. In this family the increased clinical severity from one generation to the next correlates with increased methylation of the maternal ICR1-DMR. However, all these individuals represent methylation mosaics with a gain of methylation on their maternal chromosome. Since 
the maternal ICR1-DMR in our patient and his mother is deleted an increased clinical severity could not be linked to methylation differences of this DMR on the maternal chromosome. In contrast, the hypermethylation of the IGF2-DMR0 is similar in the patient and his mother and could not explain the variable clinical expression. However, this does not exclude differences in methylation in tissues other than blood.

We report here a novel familial deletion encompassing most of the imprinted ICR1 region on chromosome 11p15. Both deletion carriers, the mother and the son, have BWS with variable clinical expression, but similar hypermethylation at IGF2 DMR0 in peripheral blood. In silico analysis revealed that two of three putative enhancer elements downstream of $H 19$ are affected by the deletion, suggesting that the remaining two enhancers are sufficient to drive IGF2 expression on the maternal chromosome.

\section{Consent}

Written informed consent was obtained from the parents for publication of this case report and any accompanying images. A copy of the written consent is available for review by the Editor of this journal.

\section{Ethics statement}

The approval was obtained from the Ethics Committee of "Aghia Sophia" Children's Hospital, Athens, Greece.

\section{Additional file}

Additional file 1: Table S1. Results of the MS-MLPA analysis. Gene dosage analysis revealed that the patient and the mother have a 50\% reduced dosage for all H19/ICR1 probes of chromosome 11p11.5 (dark grey shaded), whereas all other probes for chromosome $11 \mathrm{p} 11.5$ (light grey shaded) and all reference probes from other chromosomes showed a normal dosage. Methylation analysis showed complete methylation of approximately $100 \%$ in both the patient and his mother for the four methylation sensitive $\mathrm{H} 19$ probes, which lie inside the deletion, indicating that they have the deletion on their unmethylated maternal allele. The methylation specific probes for the ICR2 region, which is not affected by the deletion, showed a normal methylation of $~ 50 \%$. P, patient; M, mother.

\section{Competing interests}

The authors declare that they have no competing interests.

\section{Authors' contributions}

HF performed dysmorphological evaluation, genetic counseling, collected samples, initiated genetic testing and wrote the manuscript, SA first examined the patient and proposed BWS testing, CS initiated the MLPA analysis of the patient-mother-grandparents, EL was the attending physician in the Department of Pediatrics, CKG performed the complete endocrinologic evaluation, DK has established the IGF2 methylation assay and analysed the data, HJL and SB performed and analyzed CGH array analysis, FC and AR have done in silico analysis to identify enhancer elements, KB supervised this project, planned the experiments, analyzed data and wrote the manuscript. All authors reviewed and approved the final version of the manuscript.

\section{Acknowledgements}

We thank the family for participation in this study, Melanie Heitmann and Christina Lich for technical assistance, and Jasmin Beygo for helpful discussion. Part of this work was funded by the Bundesministerium für Bildung und Forschung (Network Imprinting diseases, 01 GM1114 K.B. and S.B.) and Telethon-Italia (Grant N. GGP11122 A.R.).

\section{Author details}

'Aghia Sophia Children's Hospital, University of Athens Medical School, Athens, Goudi 11527, Greece. ${ }^{2}$ Mitera Maternity Hospital, Athens, Greece. ${ }^{3}$ Institute of Human Genetics, University Hospital Essen, University Duisburg-Essen, Essen, Germany. "First Pediatric Clinic, Aghia Sophia" Children's Hospital, University of Athens School of Medicine, Athens, Greece. ${ }^{5}$ Institute of Genetics and Biophysics A. Buzzati-Traverso, CNR, DiSTABiF, 2nd University of Naples, Naples, Italy. ${ }^{6}$ Institute of Human Genetics, University Hospital Schleswig-Hostein Campus Kiel/Christian-Albrechts University Kiel, Kiel, Germany.

Received: 9 November 2014 Accepted: 22 April 2015

Published online: 06 May 2015

\section{Reference}

1. Beckwith J. Extreme cytomegaly of the adrenal fetal cortex, hyperplasia of kidneys and pancreas, and Leydig-cell hyperplasia. Another syndrome? [Abstract]. Western Society for Pediatric research: 11 November 1963, Los Angeles 1963

2. Wiedemann HR. [Familial malformation complex with umbilical hernia and macroglossia-a "New syndrome"?]. J Genet Hum. 1964;13:223-32.

3. Weksberg R, Shuman C, Beckwith JB. Beckwith-Wiedemann syndrome. Eur J Hum Genet. 2010;18:8-14.

4. Engel JR, Smallwood A, Harper A, Higgins MJ, Oshimura M, Reik W, et al. Epigenotype-phenotype correlations in Beckwith-Wiedemann syndrome. J Med Genet. 2000;37:921-6.

5. Choufani S, Shuman C, Weksberg R. Beckwith-Wiedemann syndrome. Am J Med Genet C Semin Med Genet. 2010;154C:343-54.

6. Weksberg R, Shuman C, Smith AC. Beckwith-Wiedemann syndrome. Am J Med Genet C Semin Med Genet. 2005;137C:12-23.

7. Horsthemke B. Mechanisms of imprint dysregulation. Am J Med Genet C Semin Med Genet. 2010;154C:321-8.

8. Maher ER, Reik W. Beckwith-Wiedemann syndrome: imprinting in clusters revisited. J Clin Invest. 2000;105:247-52.

9. Choufani S, Shuman C, Weksberg R. Molecular findings in Beckwith-Wiedemann syndrome. Am J Med Genet C Semin Med Genet. 2013;163C:131-40.

10. Niemitz EL, DeBaun MR, Fallon J, Murakami K, Kugoh H, Oshimura M, et al. Microdeletion of LIT1 in familial Beckwith-Wiedemann syndrome. Am J Hum Genet. 2004;75:844-9.

11. Zollino M, Orteschi D, Marangi G, De Crescenzo A, Pecile V, Riccio A, et al. A case of Beckwith-Wiedemann syndrome caused by a cryptic 11 p15 deletion encompassing the centromeric imprinted domain of the BWS locus. J Med Genet. 2010;47:429-32.

12. Algar E, Dagar V, Sebaj M, Pachter N. An 11 p15 imprinting centre region 2 deletion in a family with Beckwith Wiedemann syndrome provides insights into imprinting control at CDKN1C. PLoS One. 2011;6, e29034.

13. Gurrieri F, Zollino M, Oliva A, Pascali V, Orteschi D, Pietrobono R, et al. Mild Beckwith-Wiedemann and severe long-QT syndrome due to deletion of the imprinting center 2 on chromosome 11p. Eur J Hum Genet. 2013;21:965-9.

14. Sparago A, Cerrato F, Vernucci M, Ferrero GB, Silengo MC, Riccio A. Microdeletions in the human H19 DMR result in loss of IGF2 imprinting and Beckwith-Wiedemann syndrome. Nat Genet. 2004;36:958-60.

15. Sparago A, Russo S, Cerrato F, Ferraiuolo S, Castorina P, Selicorni A, et al. Mechanisms causing imprinting defects in familial Beckwith-Wiedemann syndrome with Wilms' tumour. Hum Mol Genet. 2007;16:254-64.

16. Demars J, Shmela ME, Rossignol S, Okabe J, Netchine I, Azzi S, et al. Analysis of the IGF2/H19 imprinting control region uncovers new genetic defects, including mutations of OCT-binding sequences, in patients with 11 p15 fetal growth disorders. Hum Mol Genet. 2010;19:803-14.

17. De Crescenzo A, Coppola F, Falco P, Bernardo I, Ausanio G, Cerrato F, et al. A novel microdeletion in the IGF2/H19 imprinting centre region defines a recurrent mutation mechanism in familial Beckwith-Wiedemann syndrome. Eur J Med Genet. 2011;54:e451-4. 
18. Demars J, Rossignol S, Netchine I, Lee KS, Shmela M, Faivre L, et al. New insights into the pathogenesis of Beckwith-Wiedemann and Silver-Russell syndromes: contribution of small copy number variations to 11 p15 imprinting defects. Hum Mutat. 2011;32:1171-82.

19. Baskin B, Choufani S, Chen YA, Shuman C, Parkinson N, Lemyre E, et al. High frequency of copy number variations (CNVs) in the chromosome 11p15 region in patients with Beckwith-Wiedemann syndrome. Hum Genet. 2014;133:321-30

20. Prawitt D, Enklaar T, Gartner-Rupprecht B, Spangenberg C, Oswald M, Lausch E, et al. Microdeletion of target sites for insulator protein CTCF in a chromosome 11 p15 imprinting center in Beckwith-Wiedemann syndrome and Wilms' tumor. Proc Natl Acad Sci U S A. 2005;102:4085-90.

21. Beygo J, Citro V, Sparago A, De Crescenzo A, Cerrato F, Heitmann M, et al. The molecular function and clinical phenotype of partial deletions of the IGF2/H19 imprinting control region depends on the spatial arrangement of the remaining CTCF-binding sites. Hum Mol Genet. 2013;22:544-57.

22. Murphy R, Baptista J, Holly J, Umpleby AM, Ellard S, Harries LW, et al. Severe intrauterine growth retardation and atypical diabetes associated with a translocation breakpoint disrupting regulation of the insulin-like growth factor 2 gene. J Clin Endocrinol Metab. 2008;93:4373-80.

23. Gronskov K, Poole RL, Hahnemann JM, Thomson J, Tumer Z, BrondumNielsen K, et al. Deletions and rearrangements of the H19/IGF2 enhancer region in patients with Silver-Russell syndrome and growth retardation. J Med Genet. 2011:48:308-11.

24. Berland S, Appelback M, Bruland O, Beygo J, Buiting K, Mackay DJ, et al. Evidence for anticipation in Beckwith-Wiedemann syndrome. Eur J Hum Genet. 2013;21:1344-8.

25. Rahmann S, Beygo J, Kanber D, Martin M, Horsthemke B, Buiting K Amplikyzer: Automated methylation analysis of amplicons from bisulfite flowgram sequencing. PeerJ PrePrints 1:e122v2 http://www.dxdoiorg/ 107287/peerjpreprints122v2 2013.

26. Frazer KA, Pachter L, Poliakov A, Rubin EM, Dubchak I. VISTA: computational tools for comparative genomics. Nucleic Acids Res. 2004;32:W273-9.

27. Blanchette M, Kent WJ, Riemer C, Elnitski L, Smit AF, Roskin KM, et al. Aligning multiple genomic sequences with the threaded blockset aligner. Genome Res. 2004;14:708-15

28. Cerrato F, De Crescenzo A, Riccio A. Looking for CDKN1C enhancers. Eur J Hum Genet. 2014;22:442-3.

29. Arney KL. H19 and Igf2-enhancing the confusion? Trends Genet. 2003;19:17-23.

30. ENCODE Project Consortium. An integrated encyclopedia of DNA elements in the human genome. Nature. 2012;489:57-74.

31. Murrell A, Ito Y, Verde G, Huddleston J, Woodfine K, Silengo MC, et al. Distinct methylation changes at the IGF2-H19 locus in congenital growth disorders and cancer. PLoS One. 2008;3, e1849.

32. Zubair M, Hilton K, Saam JR, Surani MA, Tilghman SM, Sasaki H. Structure and expression of the mouse L23mrp gene downstream of the imprinted $\mathrm{H} 19$ gene: biallelic expression and lack of interaction with the $\mathrm{H} 19$ enhancers. Genomics. 1997:45:290-6.

33. Riccio A, Sparago A, Verde G, De Crescenzo A, Citro V, Cubellis MV, et al. Inherited and Sporadic Epimutations at the IGF2-H19 locus in BeckwithWiedemann syndrome and Wilms' tumor. Endocr Dev. 2009;14:1-9.

34. Wu L, Wang L, Shangguan S, Chang S, Wang Z, Lu X, et al. Altered methylation of IGF2 DMRO is associated with neural tube defects. Mol Cell Biochem. 2013:380:33-42

\section{Submit your next manuscript to BioMed Central and take full advantage of:}

- Convenient online submission

- Thorough peer review

- No space constraints or color figure charges

- Immediate publication on acceptance

- Inclusion in PubMed, CAS, Scopus and Google Scholar

- Research which is freely available for redistribution 